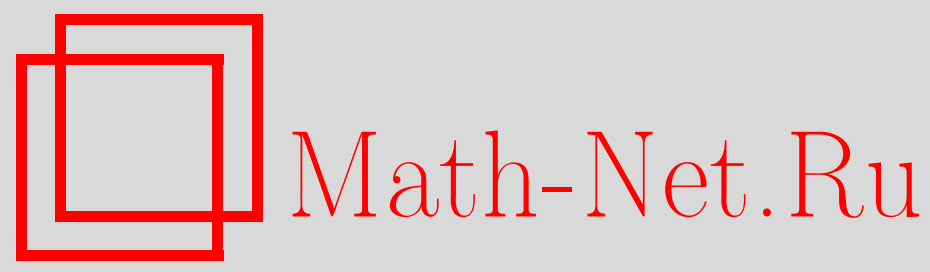

Ю. Л. Павлов, Об условных Интернет-графах, степени вершин которых не имеют математического ожидания, Дискрет. матем., 2010, том 22, выпуск 3, 20-33

DOI: https://doi.org/10.4213/dm1104

Использование Общероссийского математического портала Math-Net.Ru подразумевает, что вы прочитали и согласны с пользовательским соглашением http://www.mathnet.ru/rus/agreement

Параметры загрузки:

IP : 3.81 .55 .215

26 апреля 2023 г., $17: 47: 02$ 


\title{
Об условных Интернет-графах, степени вершин которых не имеют математического ожидания
}

\author{
(ㄷ) 2010 г. Ю. Л. Павлов
}

\begin{abstract}
Рассматриваются условные случайные графы Интернет-типа при условии, что число ребер графа известно, а степени вершин не имеют математического ожидания. Доказаны предельные теоремы для максимальной степени вершины и числа вершин заданной степени.
\end{abstract}

В работах [1-5] и многих других рассматривались Интернет-графы, которые часто используются в качестве моделей сложных сетей телекоммуникаций, включая Интернет. Такие графы содержат $N$ вершин, занумерованных числами от 1 до $N$. Степени вершин являются независимыми одинаково распределенными случайными величинами $\eta_{1}, \ldots, \eta_{N}$, такими, что

$$
\mathbf{P}\left\{\eta_{1} \geqslant k\right\}=k^{-\tau}, \quad k=1,2, \ldots,
$$

где $\tau$ - положительный параметр. Если сумма

$$
v_{N}=\eta_{1}+\ldots+\eta_{N}
$$

нечетна, то для того, чтобы сумма степеней вершин графа была четной, в граф вводится дополнительная вершина с единичной степенью. Построение Интернет-графа происходит в два этапа. На первом этапе в соответствии с распределением (1) определяются степени вершин, при этом считается, что из вершин выходят "полуребра" (см. [3]), то есть ребра, для которых смежные вершины еще не определены. Заметим, что все полуребра графа при этом различимы (занумерованы). На втором этапе образуются ребра путем равновероятного соединения полуребер друг с другом.

Получено много результатов о структуре и предельном поведении различных числовых характеристик случайных Интернет-графов при $N \rightarrow \infty$. В $[1,3]$ показано, что типичные для многих реальных сетей коммуникаций значения параметра $\tau$ распределения (1) принадлежат интервалу $(1,2)$, хотя встречаются случаи и других значений $\tau$. Замечено, что если $\tau>2$ или $\tau<1$, то свойства графов существенно отличаются от случая $\tau \in(1,2)$, следовательно, значения $\tau=1,2$ являются точками фазового перехода. Однако именно при этих значениях параметра $\tau$ поведение графа наименее изучено в силу возникающих в ходе доказательств соответствующих теорем технических трудностей. Таким образом, представляет интерес исследование Интернет-графов для всех значений $\tau$, особенно в окрестностях точек фазового перехода. При изучении большинства характеристик графов необходимо учитывать распределение суммы $v_{N}$, а также максимальной степени 
вершин и числа вершин заданной степени, поэтому в статьях $[4,5]$ основное внимание было уделено этим случайным величинам.

Далее мы будем рассматривать, как и в $[4,5]$, условные Интернет-графы при условии, что сумма степеней вершин графа $v_{N}$ известна и равна $n$. Нетрудно проверить, что

$$
\mathbf{E} \eta_{1}=\zeta(\tau)
$$

где

$$
\zeta(\tau)=\sum_{n=1}^{\infty} n^{-\tau}
$$

- значение дзета-функции Римана в точке $\tau$. Поэтому при изучении структуры и предельного поведения Интернет-графов наибольший интерес представляет зона изменения параметров $N, n$, в которой $n / N \rightarrow \zeta(\tau)$.

Обозначим $\eta_{(N)}$ и $\mu_{r}$ максимальную степень и число вершин степени $r$ соответственно. В $[4,5]$ для рассматриваемого подмножества графов изучалось предельное поведение этих случайных величин при $N, n \rightarrow \infty$. В [4] доказаны предельные теоремы в случае $\tau>0$ и $1<n / N<\zeta(\tau)$, однако при $n / N \rightarrow 1$ и $n / N \rightarrow \zeta(\tau)$ некоторые зоны изменения $N, n$ не рассмотрены. Для того, чтобы сформулировать соответствующие результаты в случае, когда $n / N \rightarrow \zeta(\tau)$ так, что $n / N<\zeta(\tau)$, обозначим через $\lambda$ решение уравнения

$$
\frac{\Phi(\lambda, \tau, 1)-(1-\lambda) \Phi(\lambda, \tau-1,1)}{1-(1-\lambda) \Phi(\lambda, \tau, 1)}=\frac{n}{N},
$$

где

$$
\Phi(\lambda, \tau, 1)=\sum_{j=0}^{\infty} \frac{\lambda^{j}}{(j+1)^{\tau}} .
$$

Из (1) следует, что

$$
p_{k}=\mathbf{P}\left\{\eta_{1}=k\right\}=k^{-\tau}-(k+1)^{-\tau}, \quad k=1,2 \ldots
$$

Введем обозначения

$$
\begin{aligned}
p_{k}(\lambda) & =\lambda^{k} p_{k}(1-(1-\lambda) \Phi(\lambda, \tau, 1))^{-1} \\
\sigma^{2}(\lambda) & =\frac{2 \Phi(\lambda, \tau-1,1)-(1-\lambda) \Phi(\lambda, \tau-2,1)-\Phi(\lambda, \tau, 1)}{1-(1-\lambda) \Phi(\lambda, \tau, 1)}-\left(\frac{n}{N}\right)^{2}, \\
\sigma_{r r}^{2}(\lambda) & =p_{r}(\lambda)\left(1-p_{r}(\lambda)-\frac{(n / N-r)^{2}}{\sigma^{2}(\lambda)} p_{r}(\lambda)\right) .
\end{aligned}
$$

Используя (3), нетрудно показать, что если $n / N \uparrow \zeta(\tau)$, то $\lambda \uparrow 1$. Ниже, в виде теорем 1 и 2, приведены результаты, полученные в [4] (см. также [5]). В этих теоремах предполагается, что выполнено одно из следующих условий:

(1) $\tau>3$

(2) $\tau=3,(\ln (1-\lambda)) / \sqrt{N} \rightarrow 0$

(3) $2<\tau<3, \sqrt{N}(1-\lambda)^{3-\tau} \rightarrow \infty$; 
(4) $\tau=2, N(1-\lambda)^{2}|\ln (1-\lambda)|^{3} \rightarrow \infty$;

(5) $0<\tau<2, N(1-\lambda)^{\tau} \rightarrow \infty$.

Теорема 1. Пусть $N, n \rightarrow \infty$ так, что $n / N \rightarrow \zeta(\tau)$. Пусть $r=r(N, n)$ выбрано так, что

$$
\frac{\tau N \lambda^{r}}{r^{\tau+1}(1-\lambda)} \rightarrow \gamma,
$$

где $\gamma$ - некоторая положительная постоянная. Тогда

$$
\mathbf{P}\left\{\eta_{(N)} \leqslant r\right\} \rightarrow e^{-\gamma}
$$

Теорема 2. Пусть $N, n \rightarrow \infty$ так, что $n / N \rightarrow \zeta(\tau)$, u $r$ - фиксированное натуральное число. Тогда для ичельх неотрицательных $k$

$$
\mathbf{P}\left\{\mu_{r}=k\right\}=\left(\sigma_{r r}(\lambda) \sqrt{2 \pi N}\right)^{-1} e^{-u_{r}^{2} / 2}(1+o(1)) .
$$

равномерно относительно $u_{r}=\left(k-N p_{r}(\lambda)\right) /\left(\sigma_{r r}(\lambda) \sqrt{N}\right)$ в любом фиксированном конечном интервале.

В статье [5] доказаны предельные теоремы для $\eta_{(N)}$ и $\mu_{r}$ в случае, когда $\tau>1$ и $n / N \rightarrow \zeta(\tau)$ так, что условия 1-5 нарушены. Заметим, что в этих теоремах не предполагается, что $n / N<\zeta(\tau)$.

Теорема 3. Пусть $N, n \rightarrow \infty, n / N \rightarrow \zeta(\tau), r=(N / \gamma)^{1 / \tau}(1+o(1))$, где $\gamma-$ некоторая положительная постоянная. Пусть также выполнено одно из следующих условий:

(1) $\tau>2, n-\zeta(\tau) N=O(\sqrt{N})$;

(2) $\tau=2, n-\zeta(2) N=O(\sqrt{N \ln N})$;

(3) $1<\tau<2, n-\zeta(\tau) N=O\left(n^{1 / \tau}\right)$.

Тогда

$$
\mathbf{P}\left\{\eta_{(N)} \leqslant r\right\} \rightarrow e^{-\gamma}
$$

Теорема 4. Пусть $N, n \rightarrow \infty, n-\zeta(\tau) N=o(\sqrt{N}), \tau>2, u r-$ фиксированное натуральное число. Тогда для цельх неотрищательных $k$

$$
\mathbf{P}\left\{\mu_{r}=k\right\}=\left(\sigma_{r r} \sqrt{2 \pi N}\right)^{-1} e^{-u_{r}^{2} / 2}(1+o(1))
$$

равномерно относительно $u_{r}=\left(k-N p_{r}\right) /\left(\sigma_{r r} \sqrt{N}\right)$ в любом фиксированном конечном интервале, где

$$
\begin{aligned}
\sigma_{r r}^{2} & =p_{r}\left(1-p_{r}-\frac{(n / N-r)^{2}}{\sigma^{2}} p_{r}\right), \\
\sigma^{2} & =2 \zeta(\tau-1)-\zeta(\tau)-\zeta^{2}(\tau) .
\end{aligned}
$$

Теорема 5. Пусть $N, n \rightarrow \infty$ и выполнено одно из следующих условий:

(1) $\tau=2, n-\zeta(2) N=o(\sqrt{N \ln N})$;

(2) $1<\tau<2, n-\zeta(\tau) N=o\left(N^{1 / \tau}\right)$. 
Тогда для ичельх неотрииательных $k$

$$
\mathbf{P}\left\{\mu_{r}=k\right\}=\frac{1+o(1)}{\sqrt{2 \pi N p_{r}\left(1-p_{r}\right)}} \exp \left\{-\frac{\left(k-N p_{r}\right)^{2}}{2 N p_{r}\left(1-p_{r}\right)}\right\}
$$

равномерно относительно $\left(k-N p_{r}\right) / \sqrt{N p_{r}\left(1-p_{r}\right)}$ в любом фиксированном конечном интервале.

Поскольку $\zeta(\tau)=\infty$ при $\tau \leqslant 1$, из (2) следует, что при таких $\tau$ математическое ожидание распределения (1) не существует. Таким образом, остался нерассмотренным случай $n / N \rightarrow \infty, \tau \leqslant 1$. Ниже доказаны теоремы 6-9 для этого случая. Используя результаты работ [6,7], нетрудно проверить, что если $\tau=1$ и $n /(N \ln N) \rightarrow 0$, то $N(1-\lambda) \rightarrow \infty$, и для описания предельного поведения $\eta_{N}$ и $\mu_{r}$ достаточно использовать теоремы 1 и 2. Аналогичная ситуация возникает при $\tau<1$ и $n / N^{1 / \tau} \rightarrow 0$, поскольку $N(1-\lambda)^{\tau} \rightarrow \infty$. Поэтому в теоремах 6-9 предполагается, что $n /(N \ln N) \geqslant C>0$ при $\tau=1$ и $n / N^{1 / \tau} \geqslant C>0$ при $0<\tau<1$. Справедливы следующие результаты.

Теорема 6. Пусть $\tau=1, N, n \rightarrow \infty$ так, что $n /(N \ln N) \geqslant C>0$. Тогда для любого фиксированного $z>0$

$$
\mathbf{P}\left\{\left(n-\eta_{(N)}\right) / N \leqslant z\right\} \rightarrow G(z),
$$

где $G(x)$ - функиия устойчивого распределения с показателем 1 и характеристической функиией

$$
f(t)=\exp \left\{-\frac{\pi}{2}|t|\left(1+i \frac{t}{|t|} \frac{2}{\pi} \ln |t|\right)\right\} .
$$

Теорема 7. Пусть $0<\tau<1, N, n \rightarrow \infty$ так, что $n / N^{1 / \tau} \rightarrow y$, где $y-$ некоторая положительная постоянная. Тогда для любого фиксированного $z>0$

$$
\mathbf{P}\left\{\eta_{(N)} / n \leqslant z\right\} \rightarrow g^{-1}(y) \sum_{k=0}^{\infty}(-1)^{k}(k !)^{-1} I_{k}(z y, y),
$$

где $g(x)$ - плотность устойчивого распределения с параметром $\tau$ и характеристической функиией

$$
\begin{aligned}
f_{\tau}(t) & =\exp \left\{-\Gamma(1-\tau)|t|^{\tau}\left(1+i \frac{t}{|t|} \operatorname{tg} \frac{\pi \tau}{2}\right) \cos \frac{\pi \tau}{2}\right\}, \\
I_{0}(u, v) & =g(v), \\
I_{k}(u, v) & =\int_{X_{k}(u, v)} \frac{\tau^{k} g\left(v-x_{1}-\ldots-x_{k}\right)}{\left(x_{1} \ldots x_{k}\right)^{\tau+1}} d x_{1} \ldots d x_{k}, \\
X_{k}(u, v) & =\left\{x_{i} \geqslant u, i=1, \ldots, k, x_{1}+\ldots+x_{k} \leqslant v\right\}, \quad k=1,2, \ldots
\end{aligned}
$$

Теорема 8. Пусть $0<\tau<1, N, n \rightarrow \infty$ так, что $n / N^{1 / \tau} \rightarrow \infty$. Тогда для любого фиксированного $z>0$

$$
\mathbf{P}\left\{\left(n-\eta_{(N)}\right) / N^{1 / \tau} \leqslant z\right\} \rightarrow G(z),
$$

где $G(x)$ - функиия устойчивого распределения с параметром $\tau$ и характеристической функиией (7). 
Заметим, что теорема 8 была доказана в магистерской диссертации Е. Н. Дертишниковой (Петрозаводский университет, 2009 г.). В настоящей статье приводится другое доказательство этой теоремы.

Теорема 9. Пусть $0<\tau \leqslant 1$, а $N, n \rightarrow \infty$ так, что $n /(N \ln N) \geqslant C>0$ при $\tau=1 u$ $n / N^{1 / \tau} \geqslant C>0$ при $\tau<1$. Тогда для цельх неотрицательных $k$

$$
\mathbf{P}\left\{\mu_{r}=k\right\}=\frac{1+o(1)}{\sqrt{2 \pi N p_{r}\left(1-p_{r}\right)}} e^{-u_{r}^{2} / 2}
$$

равномерно относительно $u_{r}=\left(k-N p_{r}\right) / \sqrt{N p_{r}\left(1-p_{r}\right)}$ в любом фиксированном конечном интервале.

Основным методом доказательства представленных результатов, как и в работах $[4,5]$, является обобщенная схема размещения частиц по ячейкам, введенная и исследованная В. Ф. Колчиным (см., например, [8]). Введем независимые одинаково распределенные случайные величины $\xi_{1}, \ldots, \xi_{N}$, для которых

$$
\mathbf{P}\left\{\xi_{1}=k\right\}=p_{k}=k^{-\tau}-(k+1)^{-\tau}, \quad k=1,2, \ldots
$$

Легко видеть, что распределения $\xi_{1}, \ldots, \xi_{N}$ совпадают с (1) и отличие от $\eta_{1}, \ldots, \eta_{N}$ состоит только в том, что на рассматриваемом множестве графов последние являются зависимыми, поскольку $\eta_{1}+\ldots+\eta_{N}=n$. Из (5) и (8) очевидным образом следует, что

$$
\mathbf{P}\left\{\eta_{1}=k_{1}, \ldots, \eta_{N}=k_{N}\right\}=\mathbf{P}\left\{\xi_{1}=k_{1}, \ldots, \xi_{N}=k_{N} \mid \xi_{1}+\ldots+\xi_{N}=n\right\} .
$$

Это равенство означает, что условия обобщенной схемы размещения выполнены. Ниже приводятся вспомогательные утверждения (леммы 1-8), с помощью которых далее будут доказаны теоремы 6-9.

Введем два набора вспомогательных независимых случайных величин $\xi_{1}^{(r)}, \ldots, \xi_{N}^{(r)}$ и $\tilde{\xi}_{1}^{(r)}, \ldots, \tilde{\xi}_{N}^{(r)}$ таких, что

$$
\begin{aligned}
& \mathbf{P}\left\{\xi_{i}^{(r)}=k\right\}=\mathbf{P}\left\{\xi_{i}=k \mid \xi_{i} \leqslant r\right\}, \quad k=1,2, \ldots, r, \quad i=1,2, \ldots, N, \\
& \mathbf{P}\left\{\tilde{\xi}_{i}^{(r)}=k\right\}=\mathbf{P}\left\{\xi_{i}=k \mid \xi_{i} \neq r\right\}, \quad k=1,2, \ldots, \quad i=1,2, \ldots, N
\end{aligned}
$$

Обозначим

$$
\begin{aligned}
\zeta_{N} & =\xi_{1}+\ldots+\xi_{N}, & \xi_{N}^{(r)} & =\xi_{1}^{(r)}+\ldots+\xi_{N}^{(r)}, \\
\tilde{\zeta}_{N}^{(r)} & =\tilde{\xi}_{1}^{(r)}+\ldots+\tilde{\xi}_{N}^{(r)}, & P_{r} & =\mathbf{P}\left\{\xi_{1}>r\right\} .
\end{aligned}
$$

Из равенства (9) легко вывести известные (см. [8]) свойства обобщенной схемы размещения.

Лемма 1. Справедливы равенства

$$
\begin{aligned}
& \mathbf{P}\left\{\eta_{(N)} \leqslant r\right\}=\left(1-P_{r}\right)^{N} \frac{\mathbf{P}\left\{\zeta_{N}^{(r)}=n\right\}}{\mathbf{P}\left\{\zeta_{N}=n\right\}}, \\
& \mathbf{P}\left\{\mu_{r}=k\right\}=\left(\begin{array}{c}
N \\
k
\end{array}\right) p_{r}^{k}\left(1-p_{r}\right)^{N-k} \frac{\mathbf{P}\left\{\tilde{\zeta}_{N-k}^{(r)}=n-k r\right\}}{\mathbf{P}\left\{\zeta_{N}=n\right\}} .
\end{aligned}
$$


Из леммы 1 ясно, что для доказательства теорем 6-9 достаточно знать предельное поведение вероятностей $\mathbf{P}\left\{\zeta_{N}=n\right\}, \mathbf{P}\left\{\zeta_{N}^{(r)}=n\right\}, \mathbf{P}\left\{\tilde{\zeta}_{N}^{(r)}=n-k r\right\}$, бинома $\left(1-P_{r}\right)^{N}$ и биномиальной вероятности $\left(\begin{array}{c}N \\ k\end{array}\right) p_{r}^{k}\left(1-p_{r}\right)^{N-k}$.

Лемма 2. Пусть $\tau=1, N, n \rightarrow \infty$ так, что $n /(N \ln N) \geqslant C>0$. Тогда

$$
\mathbf{P}\left\{\zeta_{N}=n\right\}=N / n^{2}(1+o(1)) .
$$

Доказательство. Мы воспользуемся идеей доказательства теоремы 4.2.3 книги [8]. Введем обозначение

$$
\alpha=\frac{1}{3 \ln (n / N)} .
$$

Нетрудно видеть, что

$$
\mathbf{P}\left\{\zeta_{N}=n\right\}=\mathbf{P}\left\{\zeta_{N}=n, \xi_{i} \leqslant n(1-\alpha), i=1, \ldots, N\right\}+N \mathbf{P}\left\{\zeta_{N}=n, \xi_{N}>n(1-\alpha)\right\} .
$$

Из (8) следует, что при $k \rightarrow \infty$

$$
p_{k}=\mathbf{P}\left\{\xi_{1}=k\right\}=\tau / k^{\tau+1}(1+o(1)),
$$

и это соотношение справедливо равномерно относительно $k$ при $n(1-\alpha) \leqslant k \leqslant n$. Поэтому при $\tau=1$

$$
\begin{aligned}
\mathbf{P}\left\{\zeta_{N}=n, \xi_{N}>n(1-\alpha)\right\} & =\sum_{k>n(1-\alpha)} \mathbf{P}\left\{\zeta_{N-1}=n-k, \xi_{N}=k\right\} \\
& =n^{-2} \mathbf{P}\left\{\zeta_{N-1} \leqslant n \alpha\right\}(1+o(1)) .
\end{aligned}
$$

Из теорем 2.1.1, 2.6.1 книги [9] следует, что при $\tau=1$ распределение $\zeta_{N} / N$ слабо сходится к устойчивому закону с показателем 1 . Из (12) и условия $n /(N \ln N) \geqslant C>0$ получаем, что $n \alpha / N \rightarrow \infty$, следовательно,

$$
\mathbf{P}\left\{\zeta_{N-1} \leqslant n \alpha\right\} \rightarrow 1 .
$$

Отсюда и из (15) находим, что

$$
\mathbf{P}\left\{\zeta_{N}=n, \xi_{N}>n(1-\alpha)\right\}=n^{-2}(1+o(1)) .
$$

Рассмотрим сумму

$$
R(\omega)=\sum_{k \leqslant n(1-\alpha)} p_{k} \exp \{\omega k\} .
$$

Из (8) следует, что для натуральных $l$ и любом $\tau>0$

$$
\sum_{k>l} p_{k}=(l+1)^{-\tau},
$$

а при $\tau=1$

$$
\sum_{k \leqslant l} k p_{k} \leqslant C_{1} \ln l
$$


здесь и далее во всех доказательствах символы $C_{1}, C_{2}, \ldots$ означают некоторые положительные постоянные. При $x \rightarrow 0$ справедливо соотношение

$$
e^{x}=1+O(x)
$$

поэтому из (17)-(19) и условия $n /(N \ln N) \geqslant C>0$ находим, что

$$
R(1 /(n(1-\alpha)))=1+O((\ln n) / n)=1+O\left(N^{-1}\right) .
$$

Введем вспомогательные независимые одинаково распределенные случайные величины $\xi_{1}(\alpha), \ldots, \xi_{N}(\alpha)$ такие, что

$$
\mathbf{P}\left\{\xi_{1}(\alpha)=k\right\}=\exp \{k /(n(1-\alpha))\} p_{k} / R(1 /(n(1-\alpha))), \quad k \leqslant n(1-\alpha) .
$$

Обозначим

$$
\zeta_{N}(\alpha)=\xi_{1}(\alpha)+\ldots+\xi_{N}(\alpha)
$$

Легко видеть, что

$$
\mathbf{P}\left\{\zeta_{N}=n, \xi_{i} \leqslant n(1-\alpha), i=1, \ldots, N\right\}=R^{N}(1 /(n(1-\alpha))) e^{-1 / \alpha} \mathbf{P}\left\{\zeta_{N}(\alpha)=n\right\} .
$$

Докажем, что при достаточно больших $n$

$$
\mathbf{P}\left\{\zeta_{N}(\alpha)=n\right\} \leqslant C_{2} N^{-1} .
$$

Обозначим $\varphi^{(\alpha)}(t)$ характеристическую функцию случайной величины $\xi_{1}(\alpha)$. По формуле обращения

$$
\mathbf{P}\left\{\zeta_{N}(\alpha)=n\right\}=\frac{1}{2 \pi N} \int_{-\pi N}^{\pi N} \exp \left\{-\frac{i t n}{N}\right\}\left(\varphi^{(\alpha)}\left(\frac{t}{N}\right)\right)^{N} d t .
$$

Рассмотрим выражение

$$
\left(\varphi^{(\alpha)}(t)\right)^{N}=\left(\frac{R(1 /(n(1-\alpha))+i t)}{R(1 /(n(1-\alpha)))}\right)^{N} .
$$

Если $k \leqslant n(1-\alpha)$, то

$$
\exp \{k /(n(1-\alpha))\}=1+\delta k / n, \quad \delta<2,
$$

поэтому

$$
R(1 /(n(1-\alpha))+i t)=\sum_{k \leqslant n(1-\alpha)} p_{k} e^{i t k}+n^{-1} \sum_{k \leqslant n(1-\alpha)} \delta k p_{k} e^{i t k}
$$

и из (18), (19), (20), (24) находим, что

$$
\left|\varphi^{(\alpha)}\right|^{N} \leqslant C_{3}|\varphi(t)|^{N},
$$

где $\varphi(t)-$ характеристическая функция случайной величины $\xi_{1}$. Используя (8), нетрудно получить, что при всех $\tau$

$$
\varphi(t)=1+\left(e^{i t}-1\right) \Phi\left(e^{i t}, \tau, 1\right),
$$


где функция $\Phi\left(e^{i t}, \tau, 1\right)$ определена в (4). Из полученных в $[6,7]$ результатов следует, что при $t \rightarrow 0$

$$
\Phi\left(e^{i t}, 1,1\right)=-(1-i t) \ln (-i t)-i t / 2+O\left(t^{2} \ln t\right),
$$

поэтому из (26) получаем, что

$$
\varphi(t)=1-i t \ln t-\left(t^{2} \ln t\right) / 2+O\left(t^{2}\right) .
$$

Учитывая, что при $0<\varepsilon \leqslant t \leqslant \pi$ для любой характеристической функции $\psi(t)$ решетчатого распределения с единичным максимальным шагом справедливо неравенство

$$
|\psi(t)| \leqslant e^{-C_{4}}
$$

из (23), (25) и (27) находим, что

$$
\begin{aligned}
\mathbf{P}\left\{\zeta_{N}(\alpha)=n\right\}= & \frac{1}{2 \pi N} \int_{|t|<\varepsilon N} \exp \left\{-\frac{i t n}{N}\right\}\left(\varphi^{(\alpha)}\left(\frac{t}{N}\right)\right)^{N} d t \\
& \quad+\frac{1}{2 \pi N} \int_{\varepsilon N \leqslant|t| \leqslant \pi N} \exp \left\{-\frac{i t n}{N}\right\}\left(\varphi^{(\alpha)}\left(\frac{t}{N}\right)\right)^{N} d t \\
& <\frac{1}{2 \pi N}\left(\int_{0}^{\infty} e^{-t^{2}} d t+2 \pi N e^{-C_{4} N}\right),
\end{aligned}
$$

откуда и следует (22).

Из (12) и (20)-(22) вытекает, что

$$
\mathbf{P}\left\{\zeta_{N}=n, \xi_{i} \leqslant n(1-\alpha), i=1, \ldots, N\right\} \leqslant C_{2} N^{2} / n^{3}=o\left(N / n^{2}\right),
$$

поэтому из (13) и (16) получаем утверждение леммы 2.

Из теорем 2.2.2, 2.6.1 и 4.2.1 книги [9] следует такое утверждение.

Лемма 3. Пусть $0<\tau<1, N \rightarrow \infty$. Тогда

$$
\sup _{k}\left|N^{1 / \tau} \mathbf{P}\left\{\zeta_{N}=k\right\}-g\left(k / N^{1 / \tau}\right)\right| \rightarrow 0,
$$

где $g(x)$ - плотность устойчивого распределения с показателем $\tau$ и характеристической функцией (7).

Рассмотрим теперь предельное поведение вероятности $\mathbf{P}\left\{\zeta_{N}=n\right\}$ при $0<\tau<1$ в области больших уклонений.

Лемма 4. Пусть $0<\tau<1, N, n \rightarrow \infty$ так, что $n / N^{1 / \tau} \rightarrow \infty$. Тогда

$$
\mathbf{P}\left\{\zeta_{N}=n\right\}=\tau N n^{-(\tau+1)}(1+o(1))
$$

Доказательство. Мы будем следовать схеме доказательства леммы 2. Обозначим

$$
\alpha=\frac{N^{2}}{n^{\tau+1}} .
$$

Из (14) находим, по аналогии с (15), что

$$
\mathbf{P}\left\{\zeta_{N}=n, \xi_{N} \geqslant n(1-\alpha)\right\}=\tau n^{-(\tau+1)} \mathbf{P}\left\{\zeta_{N-1} \leqslant n \alpha\right\}(1+o(1)) .
$$


Из (30) и леммы 3 следует, что $n \alpha / N^{1 / \tau} \rightarrow \infty$, поэтому $\mathbf{P}\left\{\zeta_{N-1} \leqslant n \alpha\right\} \rightarrow 1$ и, согласно (31),

$$
\mathbf{P}\left\{\zeta_{N}=n, \xi_{N} \geqslant n(1-\alpha)\right\}=\tau n^{-(\tau+1)}(1+o(1)) .
$$

Легко проверить, что в условиях леммы 4 равенство (20) остается верным, при этом вместо (19) можно использовать оценку

$$
\sum_{k \leqslant l} k p_{k} \leqslant C_{1} l^{1-\tau}
$$

Вводя случайные величины $\xi_{1}(\alpha), \ldots, \xi_{N}(\alpha)$, как и при доказательстве леммы 2, убеждаемся в справедливости соотношений (21), (23)-(25). Используя известную (см. [7]) асимптотику функции (4) при $z \rightarrow 1$ и $0<\tau<1$

$$
\Phi(z, \tau, 1)=\Gamma(1-\tau)(1-z)^{\tau-1}(1+o(1)),
$$

из (25), (26) находим, что при $t \rightarrow 0$

$$
\left|\varphi^{(\alpha)}(t)\right| \leqslant \exp \left\{-C_{1}|t|^{\tau}\right\} .
$$

Учитывая это неравенство, как и в лемме 2, нетрудно получить оценку типа (22). Поэтому из (20), (21) и (30) находим, что

$$
\mathbf{P}\left\{\zeta_{N}=n, \xi_{i} \leqslant n(1-\alpha), i=1, \ldots, N\right\}=o\left(N n^{-(\tau+1)}\right) .
$$

Отсюда и из (13), (32) следует справедливость леммы 4.

Далее описывается асимптотика сумм $\zeta_{N}^{(r)}$ и $\tilde{\zeta}_{N}^{(r)}$.

Лемма 5. Пусть $\tau=1, N, n \rightarrow \infty$ так, что $n /(N \ln N) \geqslant C>0, r=n-z N$, где $z-$ некоторая положительная постоянная. Тогда

$$
\mathbf{P}\left\{\zeta_{N}^{(r)}=n\right\}=N n^{-2} \int_{z}^{\infty} g(x) d x(1+o(1))
$$

где $g(x)$ - плотность устойчивого распределения с показателем 1 и характеристической функиией (6).

Доказательство. Очевидно, что справедливо равенство, аналогичное (13):

$\mathbf{P}\left\{\zeta_{N}^{(r)}=n\right\}=\mathbf{P}\left\{\zeta_{N}^{(r)}=n, \xi_{i}^{(r)} \leqslant n(1-\alpha), i=1, \ldots, N\right\}+N \mathbf{P}\left\{\zeta_{N}^{(r)}=n, \xi_{N}^{(r)}>n(1-\alpha)\right\}$,

где величина $\alpha$ определена в (12). Из (8), (10), (14) и (18) следует, что при $\tau=1$ и $k \rightarrow \infty$

$$
\mathbf{P}\left\{\xi_{1}^{(r)}=k\right\}=p_{k} /\left(1-P_{r}\right)=k^{-2}(1+o(1)),
$$

поэтому

$$
\begin{aligned}
\mathbf{P}\left\{\zeta_{N}^{(r)}=n, \xi_{N}^{(r)} \geqslant n(1-\alpha)\right\} & =\sum_{n(1-\alpha) \leqslant k \leqslant r} \mathbf{P}\left\{\zeta_{N-1}^{(r)}=n-k, \xi_{N}^{(r)}=k\right\} \\
& =n^{-2} \mathbf{P}\left\{n-r \leqslant \zeta_{N-1}^{(r)} \leqslant n \alpha\right\} .
\end{aligned}
$$


Обозначим $\varphi_{r}(t)$ характеристическую функцию случайной величины $\xi_{1}^{(r)}$, тогда

$$
\varphi_{r}(t)=\frac{1}{1-P_{r}}\left(\varphi(t)-\sum_{k=r+1}^{\infty} p_{k} e^{i t k}\right) .
$$

В силу (8),

$$
\left|\sum_{k=r+1}^{\infty} p_{k} e^{i t k}\right| \leqslant P_{r}=r^{-1}(1+o(1))=o\left(N^{-1}\right),
$$

поэтому из (36) получаем, что

$$
\varphi_{r}^{N-1}(t / N)=\varphi^{N-1}(t / N)(1+o(1)) .
$$

Отсюда, из леммы 3 и (35) следует, что

$$
\mathbf{P}\left\{\zeta_{N}^{(r)}=n, \xi_{N}^{(r)} \geqslant n(1-\alpha)\right\}=n^{-2} \int_{z}^{\infty} g(x) d x(1+o(1)) .
$$

Учитывая условие $n /(N \ln N) \geqslant C>0$, нетрудно получить, аналогично (29), что

$$
\mathbf{P}\left\{\zeta_{N}^{(r)}=n, \xi_{i}^{(r)} \leqslant n(1-\alpha), i=1, \ldots, N\right\}=o\left(N / n^{2}\right),
$$

поэтому из (34) и (37) следует справедливость леммы 5.

Лемма 6. Пусть $0<\tau<1, N, n \rightarrow \infty$ так, что $n / N^{1 / \tau} \rightarrow y$, где y-некоторая положительная постоянная. Пусть $v=j / N^{1 / \tau}$, где $j-$ натуральное число. Если $r=z n+O(1)$, где z-фиксированное положительное число, то

$$
N^{1 / \tau} \mathbf{P}\left\{\zeta_{N}^{(r)} / N^{1 / \tau}=v\right\} \rightarrow e^{E(0, z)} \sum_{k=0}^{\infty}(-1)^{k}(k !)^{-1} I_{k}(z y, v)
$$

равномерно относительно $v, 0<v_{0} \leqslant v \leqslant v_{1}$, для любых конечных $v_{0}, v_{1}$, где

$$
E(t, z)=\tau \int_{z y}^{\infty} x^{-(\tau+1)} e^{i t x} d x
$$

а интеграль $I_{k}(u, v)$ определень в теореме 7.

Доказательство. Обозначим $\Psi_{r}(t)$ характеристическую функцию случайной величины $\zeta_{N}^{(r)} / N^{1 / \tau}$. Из (8), (10) и (26) следует, что

$$
\Psi_{r}(t)=\left(1-P_{r}\right)^{-N}\left(\varphi\left(t / N^{1 / \tau}\right)-\sum_{k=r+1}^{\infty} p_{k} \exp \left\{i t k / N^{1 / \tau}\right\}\right)^{N}
$$

Отсюда и из леммы 3 получаем, что при любом фиксированном $t$

$$
\Psi_{r}(t)=\left(1-P_{r}\right)^{-N} f_{\tau}(t)\left(1-(1+o(1)) \sum_{k=r+1}^{\infty} p_{k} \exp \left\{i t k / N^{1 / \tau}\right\}\right)^{N}
$$


где характеристическая функция $f_{\tau}(t)$ определена в (7). Используя (14), нетрудно получить, что

$$
\sum_{k=r+1}^{\infty} p_{k} \exp \left\{i t k / N^{1 / \tau}\right\}=N^{-1} E(t, z)(1+o(1)) .
$$

Полагая в (40) $t=0$, из (39) находим, что

$$
\Psi_{r}(t)=f_{\tau}(t) \exp \{-E(t, z)+E(0, z)\}+o(1) .
$$

Поскольку $E(t, z)$ является преобразованием Фурье функции $t^{-(\tau+1)}$, разлагая $\exp \{-E(t, z)\}$ в ряд по степеням $E(t, z)$ и учитывая, что $f_{\tau}(t)$ является характеристической функцией устойчивого закона с показателем $\tau$, имеющего плотность $g(x)$ (см. теорему 7), находим, что $\Psi_{r}(t)$ сходится к характеристической функции распределения с плотностью

$$
q(x)=e^{E(0, z)} \sum_{k=0}^{\infty}(-1)^{k}(k !)^{-1} I_{k}(z y, x) .
$$

По формуле обращения представим вероятность $\mathbf{P}\left\{\zeta_{N}^{(r)}=j\right\}$ в виде

$$
\mathbf{P}\left\{\zeta_{N}^{(r)} / N^{1 / \tau}=v\right\}=\left(2 \pi N^{1 / \tau}\right)^{-1} \int_{-\pi N^{1 / \tau}}^{\pi N^{1 / \tau}} e^{-i v t} \Psi_{r}(t) d t .
$$

Из (7) ясно, что

$$
\left|f_{\tau}(t) \exp \{-E(t, z)+E(0, z)\}\right| \leqslant C_{1} \exp \left\{-C_{2}|t|^{\tau}\right\} .
$$

Отсюда следует, что $f_{\tau}(t) \exp \{-E(t, z)+E(0, z)\}$ интегрируема по $t$ и для плотности (42) мы можем применить формулу обращения:

$$
q(v)=(2 \pi)^{-1} \int_{-\infty}^{\infty} e^{-i v t} f_{\tau}(t) \exp \{-E(t, z)+E(0, z)\} d t
$$

поэтому из (43) находим, что

$$
2 \pi N^{1 / \tau} \mathbf{P}\left\{\zeta_{N}^{(r)} / N^{1 / \tau}=v\right\}-2 \pi q(v)=I_{1}+I_{2}+I_{3}+I_{4},
$$

где

$$
\begin{aligned}
& I_{1}=\int_{-A}^{A} e^{-i t v}\left(\Psi_{r}(t)-f_{\tau}(t) \exp \{-E(t, z)+E(0, z)\}\right) d t, \\
& I_{2}=\int_{A<|t| \leqslant \varepsilon N^{1 / \tau}} e^{-i t v} \Psi_{r}(t) d t, \\
& I_{3}=\int_{\varepsilon N^{1 / \tau}<|t| \leqslant \pi N^{1 / \tau}} e^{-i t v} \Psi_{r}(t) d t, \\
& I_{4}=-\int_{A<|t|} f_{\tau}(t) \exp \{-i t v-E(t, z)+E(0, z)\} d t ;
\end{aligned}
$$

положительные постоянные $A$ и $\varepsilon$ будут выбраны позднее. 
Очевидно, что для доказательства леммы достаточно показать, что каждый из интегралов $I_{1}, I_{2}, I_{3}, I_{4}$ может быть сделан сколь угодно малым выбором достаточно больших $N, n$. Из (41) следует, что $I_{1} \rightarrow 0$. Используя (44), получаем неравенство

$$
\left|I_{4}\right| \leqslant 2 C_{1} \int_{A}^{\infty} e^{-C_{2}|t|^{\tau}} d t
$$

а последнее выражение может быть сделано сколь угодно малым выбором достаточно большого $A$. Из (26) и (33) нетрудно найти, что если $|t| \leqslant \varepsilon$ и $\varepsilon$ достаточно мало, то

$$
|\varphi(t)| \leqslant \exp \left\{-C_{3}|t|^{\tau}\right\}
$$

Отсюда и из (39) следует, что при достаточно больших $N$

$$
\left|I_{2}\right| \leqslant 2\left(\frac{1+2 P_{r}}{1-P_{r}}\right)^{N} \int_{A}^{\infty} e^{-C_{3}|t|^{\tau}} d t
$$

поэтому из (40) получаем, что

$$
\left|I_{2}\right| \leqslant C_{4} \int_{A}^{\infty} e^{-C_{3}|t|^{\tau}} d t
$$

а этот интеграл оценивается так же, как и $I_{4}$. Наконец, используя (28) и (38), находим, чTO

$$
\left|I_{3}\right| \leqslant C_{5} N^{1 / \tau}\left(\frac{e^{-C_{6}}+P_{r}}{1-P_{r}}\right)^{N} \rightarrow 0 .
$$

Лемма 6 доказана.

Следуя доказательствам лемм 4, 5 и задавая $\alpha$ по формуле (30) и учитывая (31), (35), получаем такой результат.

Лемма 7. Пусть $0<\tau<1, N, n \rightarrow \infty$ так, что $n / N^{1 / \tau} \rightarrow \infty, r=n-z N^{1 / \tau}$, где $z-$ фиксированное положительное число. Тогда

$$
\mathbf{P}\left\{\zeta_{N}^{(r)}=n\right\}=\tau N n^{-(\tau+1)} \int_{z}^{\infty} g(x) d x(1+o(1))
$$

где $g(x)$ - плотность устойчивого распределения с показателем $\tau$ и характеристической функиией (7).

Лемма 8. Пусть $0<\tau \leqslant 1, N, n \rightarrow \infty$ так, что $n /(N \ln N) \geqslant C>0$ при $\tau=1 u$ $n / N^{1 / \tau} \geqslant C>0$ при $\tau<1$. Пусть $r-$ фиксированное натуральное число,

$$
S=N\left(1-p_{r}\right)(1+o(1))
$$

Тогда

$$
\mathbf{P}\left\{\tilde{\zeta}_{S}^{(r)}=n\right\}=\tau N n^{-(\tau+1)}(1+o(1))
$$


Доказательство. Аналогично (13) получаем,что

$$
\mathbf{P}\left\{\tilde{\zeta}_{S}^{(r)}=n\right\}=\mathbf{P}\left\{\tilde{\zeta}_{S}^{(r)}=n, \tilde{\xi}_{i}^{(r)} \leqslant n(1-\alpha), i=1, \ldots, S\right\}+S \mathbf{P}\left\{\tilde{\zeta}_{S}^{(r)}=n, \tilde{\xi}_{S}^{(r)}>n(1-\alpha)\right\},
$$

где $\alpha$ задается равенством (12) при $\tau=1$ и равенством (30) в случае $\tau<1$. Из (11) и (14) следует, что при $k \rightarrow \infty$

$$
\mathbf{P}\left\{\tilde{\xi}_{1}^{(r)}=k\right\}=\tau\left(k^{\tau+1}\left(1-p_{r}\right)\right)^{-1}(1+o(1)),
$$

откуда находим, аналогично (15), (16), что

$$
\mathbf{P}\left\{\tilde{\zeta}_{S}^{(r)}=n, \tilde{\xi}_{S}^{(r)}>n(1-\alpha)\right\}=\tau\left(n^{\tau+1}\left(1-p_{r}\right)\right)^{-1}(1+o(1)) .
$$

Оценка вероятности $\mathbf{P}\left\{\tilde{\zeta}_{S}^{(r)}=n, \tilde{\xi}_{i}^{(r)} \leqslant n(1-\alpha), i=1, \ldots, S\right\}$ проводится так же, как и в (29), поэтому из (45), (46) следует справедливость леммы 8.

Теперь мы можем доказать теоремы 6-9. Из (18) следует, что в условиях теорем 6, 8 при $r=n-z N, 0<z<\infty$,

$$
\left(1-P_{r}\right)^{N} \rightarrow 0
$$

Отсюда и из лемм 1, 2, 5 получаем утверждение теоремы 6. Теорему 7 легко доказать, полагая $r=z n+O(1)$ и используя леммы $1,3,6$ и (40), а теорема 8 вытекает из лемм 1, 4, 7 и (47).

В условиях теоремы 9 положим $S=N-k$, где

$$
k=N p_{r}+u_{r} \sqrt{N p_{r}\left(1-p_{r}\right)} .
$$

Тогда из леммы 8 находим, что при $\tau=1$ справедливо соотношение

$$
\mathbf{P}\left\{\tilde{\zeta}_{N-k}^{(r)}=n-k r\right\}=N / n^{2}(1+o(1))
$$

поэтому из леммы 2 следует, что

$$
\mathbf{P}\left\{\tilde{\zeta}_{N-k}^{(r)}=n-k r\right\} / \mathbf{P}\left\{\zeta_{N}=n\right\} \rightarrow 1 .
$$

Пусть $0<\tau<1, n / N^{1 / \tau} \rightarrow y, 0<y<\infty$. Нетрудно видеть, что для сумм $\tilde{\zeta}_{N-k}^{(r)}$ из теорем 2.2.2, 2.6.1, 4.2.1 из [9] вытекает утверждение, аналогичное лемме 3, следовательHO,

$$
N^{1 / \tau} \mathbf{P}\left\{\tilde{\zeta}_{N-k}^{(r)}=n-k r\right\}=g(y)(1+o(1)),
$$

поэтому соотношение (48) сохраняет силу. Если же $n / N^{1 / \tau} \rightarrow \infty$, то (48) следует из лемм 4 и 8. Согласно нормальному приближению биномиального распределения,

$$
\left(\begin{array}{l}
N \\
k
\end{array}\right) p_{r}^{k}\left(1-p_{r}\right)^{N-k}=\frac{1+o(1)}{\sqrt{2 \pi N p_{r}\left(1-p_{r}\right)}} e^{-u_{r}^{2} / 2}
$$

равномерно относительно $u_{r}$ в любом фиксированном конечном интервале, поэтому теорема 9 следует из леммы 1 и (48). 


\section{Список литературы}

1. Faloutsos C., Faloutsos P., Faloutsos M., On power-law relationships of the Internet topology. Computer Communications Rev. (1999) 29, 251-262.

2. Janson S., Luczak T., Rucinski A., Random graphs. Wiley, New York, 2000.

3. Reittu H., Norros I., On the power-law random graph model of massive data networks. Performance Evaluation (2004) 55, 3-23.

4. Павлов Ю. Л., Чеплюкова И. А., Случайные графы Интернет-типа и обобщенная схема размещения. Дискретная математика (2008) 20, №3, 3-18.

5. Павлов Ю. Л., О предельных распределениях степеней вершин в условных Интернет-графах. Дискретная математика (2009) 21, №3, 14-23.

6. Robinson J. E., Note on the Bose-Einstein integral functions. Phys. Rev. 2 (1951) 83, 678-679.

7. Wood D., Techn. Rep. 15-20, Univ. Kent, 1992.

8. Колчин В. Ф., Случайные графы. Физматлит, Москва, 2000.

9. Ибрагимов И. А., Линник Ю. В., Независимьле и стаџионарно связанные величины. Наука, Москва, 1965.

Статья поступила 1.04.2009. 